\title{
Universiteit
}

Leiden

The Netherlands

\section{Relatively high risk for hepatocellular carcinoma in patients with primary biliary cirrhosis not responding to ursodeoxycholic acid}

Kuiper, E.M.M.; Hansen, B.E.; Adang, R.P.R.; Nieuwkerk, C.M.J. van; Timmer, R.; Drenth, J.P.H.; ... ; Dutch PBC Study Grp

\section{Citation}

Kuiper, E. M. M., Hansen, B. E., Adang, R. P. R., Nieuwkerk, C. M. J. van, Timmer, R., Drenth, J. P. H., ... Buuren, H. R. van. (2010). Relatively high risk for hepatocellular carcinoma in patients with primary biliary cirrhosis not responding to ursodeoxycholic acid. European Journal of Gastroenterology And Hepatology, 22(12), 1495-1502. doi:10.1097/MEG.0b013e32834059e7

Version: $\quad$ Not Applicable (or Unknown)

License: $\quad$ Leiden University Non-exclusive license

Downloaded from: $\quad$ https://hdl.handle.net/1887/117390

Note: To cite this publication please use the final published version (if applicable). 


\section{Relatively high risk for hepatocellular carcinoma in patients with primary biliary cirrhosis not responding to ursodeoxycholic acid}

Edith M.M. Kuiper ${ }^{\mathrm{a}}$, Bettina E. Hansen ${ }^{\mathrm{a}, \mathrm{b}}$, Rob P.R. Adang ${ }^{\mathrm{c}}$, Carin M.J. van Nieuwkerk ${ }^{d}$, Robin Timmer ${ }^{\mathrm{e}}$, Joost P.H. Drenth, ${ }^{f}$ Piet Spoelstra ${ }^{\mathrm{g}}$, Hans T. Brouwer ${ }^{h}$, Johan P.H. Kuyvenhoven ${ }^{i}$ and Henk R. van Buuren ${ }^{a}$; for the Dutch PBC Study Group

\begin{abstract}
Background The reported incidence of hepatocellular carcinoma (HCC) among patients with primary biliary cirrhosis (PBC) varies from $0.7-3.8 \%$, whereas in cirrhotic patients the risk is considerably higher. Age, male sex, cirrhosis, and portal hypertension are reported risk factors. It has been suggested that ursodeoxycholic acid (UDCA) may protect against HCC. We aimed to define risk factors for the development of $\mathrm{HCC}$ at the time of PBC diagnosis and to identify, among patients treated with UDCA for a long term, a subgroup that could benefit from screening.
\end{abstract}

Methods Prospective multicenter cohort study of patients with established PBC treated with $13-15 \mathrm{mg} / \mathrm{kg} /$ day UDCA. Age, sex, antimitochondrial antibodies, bilirubin, albumin, alkaline phosphatase, alanine aminotransferase, aspartate amino transferase, cirrhosis, portal hypertension, Mayo Risk Score, prognostic class (based on bilirubin and albumin levels), and response to UDCA (normalization of bilirubin and/or albumin levels) were analyzed as potential risk factors in Cox regression analysis.

Results Three hundred and seventy-five patients were included, median follow-up was 9.7 years. HCC occurred in nine patients, corresponding with an annual incidence of $0.2 \%$. The factor significantly associated with the development of HCC was the response to UDCA $(P<0.001)$. The risk for HCC was highest in the group

\section{Introduction}

Primary biliary cirrhosis (PBC) is a chronic cholestatic liver disease, which predominantly occurs in middle-aged women. In patients with nonadvanced disease and who are treated with ursodeoxycholic acid survival seems to be comparable with the general population and causes of death are mainly nonliver related [1]. The overall incidence of hepatocellular carcinoma (HCC) among PBC patients varies from 0.7 to $3.8 \%$ [2,3], whereas an incidence of up to $14 \%$ has been reported for those with cirrhotic disease (Table 1) [3]. Limited data are available on a possible protective effect of treatment with ursodeoxycholic acid (UDCA) [8].

Several risk factors for HCG have been reported, including histological stage, sex, age, presence of portal of nonresponders to UDCA: the 10 years incidence of HCC was $9 \%$ and the 15 years incidence was $20 \%$. The number needed to screen in this subgroup was 11 .

Conclusion In UDCA treated PBC patients the risk of HCC is relatively low. The main risk factor for HCC in this study was the absence of biochemical response to UDCA after 1 -year treatment. Eur $J$ Gastroenterol Hepatol 22:1495-1502 @ 2010 Wolters Kluwer Health | Lippincott Williams \& Wilkins.

European Journal of Gastroenterology \& Hepatology 2010, 22:1495-1502

Keywords: hepatocellular carcinoma, primary biliary cirrhosis, risk factors, screening

Departments of a Gastroenterology and Hepatology, ${ }^{b}$ Epidemiology and Biostatistics, Erasmus University Medical Center, Rotterdam, ${ }^{\mathrm{C}}$ Department of Gastroenterology and Hepatology, VieCuri Medical Center, Venlo, ${ }^{\mathrm{d}}$ Department of Gastroenterology and Hepatology, VU University Hospital Amsterdam, Amsterdam, ${ }^{e}$ Department of Gastroenterology and Hepatology, St Antonius Hospital, Nieuwegein, 'Department of Gastroenterology and Hepatology, Radboud University Hospital Nijmegen, Nijmegen, ${ }^{9}$ Department of Gastroenterology and Hepatology, Medical Center Leeuwarden, Leeuwarden, hDepartment of Gastroenterology and Hepatology, Reinier de Graaf Gasthuis, Delft and 'Department of Gastroenterology and Hepatology, Kennemer Gasthuis, Haarlem, The Netherlands

Correspondence to Henk R. van Buuren, MD, PhD, Department of Gastroenterology and Hepatology, Erasmus Medical Center Rotterdam, PO Box 2040, 3000 CA Rotterdam, The Netherlands Tel: +31 10704 2261; fax: + 31104365916 ; e-mail: h.vanbuuren@erasmusmc.nl

Received 13 June 2010 Accepted 31 August 2010

hypertension, and history of blood transfusion [3,8,11]. Ideally, surveillance programs should be limited to those patients carrying a significant risk for HCC and these patients should be easily identifiable to prevent burden of medical resources. In addition, tumors should be detected at an early stage.

Today, surveillance for HCG is solely recommended for PBC patients with cirrhosis [12]. This strategy, however, has inherent disadvantages, the most important being the necessity of repeated diagnostic procedures, such as liver biopsy, in the majority of cases who present with noncirrhotic disease. Further, liver biopsy is associated with sampling error and interpretation difficulties. Silveira $e t$ al. [11] developed a model to predict HCG 
Table 1 Reported annual and cumulative incidences of hepatocellular carcinoma in patients with primary biliary cirrhosis

\begin{tabular}{|c|c|c|c|c|c|c|c|c|c|}
\hline \multirow[b]{2}{*}{ Year } & \multirow[b]{2}{*}{ First author } & \multirow[b]{2}{*}{ Time period } & \multirow{2}{*}{$\begin{array}{c}\text { Patients } \\
\text { N }\end{array}$} & \multirow[b]{2}{*}{ UDCA } & \multirow{2}{*}{$\begin{array}{c}\text { Follow-up } \\
\text { Years }\end{array}$} & \multicolumn{2}{|c|}{ Annual incidence } & \multicolumn{2}{|c|}{ Cumulative incidence } \\
\hline & & & & & & Total (\%) & $\begin{array}{l}\text { Ludwig stage } \\
\text { III/IV }^{\mathrm{a}}(\%)\end{array}$ & Total (\%) & $\begin{array}{l}\text { Ludwig stage } \\
\text { III/IV }^{\mathrm{a}}(\%)\end{array}$ \\
\hline 1994 & Farinati et al. [4] & $1976-1981$ & 89 & b & $5.5 / 4.3$ & 0.4 & 1.4 & 2.2 & 6.1 \\
\hline 1997 & Jones et al. [5] & $1975-1995$ & 667 & $\mathrm{~b}$ & 7.3 & 0.3 & 0.8 & 2.4 & 5.9 \\
\hline 1999 & Nijhawan et al. [6] & $1976-1985$ & 1689 & $\mathrm{~b}$ & 4.4 & 0.2 & - & 0.7 & - \\
\hline 2001 & Caballeria et al. [7] & 1977-1996 & 140 & $b$ & 5.6 & 0.6 & 2.0 & 3.6 & 11.1 \\
\hline 2001 & Shibuya et al. [8] & $1980-1998$ & 396 & $\mathrm{~b}$ & 3.6 & 1.0 & 3.4 & 3.5 & 12.3 \\
\hline 2002 & Findor et al. [9] & 1978-1998 & 292 & $\mathrm{~b}$ & 3.0 & 0.5 & 1.1 & 1.3 & 3.4 \\
\hline 2003 & Floreani et al. [10] & $1973-1999$ & 170 & $\mathrm{~b}$ & 6.2 & 0.5 & - & 3.0 & - \\
\hline 2007 & Jackson et al. [2] & 1987-2002 & 395 & Yes & 3.7 & 0.2 & - & 0.7 & - \\
\hline 2008 & Deutsch et al. [3] & $1987-2005$ & 212 & Partly & $6.0 / 7.0$ & 0.6 & 2.1 & 3.8 & 14.5 \\
\hline This study & & 1990-2007 & 375 & Yes & 9.7 & 0.2 & 0.2 & 2.4 & 1.9 \\
\hline
\end{tabular}

UDCA, ursodeoxycholic acid.

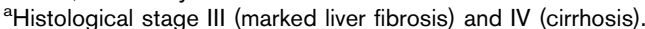

${ }^{b}$ No details on UDCA treatment.

based on age, sex, portal hypertension status, and history of blood transfusions, but the criterion of portal hypertension has the same disadvantages as histological status and the model has not been validated yet.

The aim of this study was to evaluate epidemiology of PBC related mortality focused on HCC in a large UDCAtreated cohort. We aimed to define risk factors for the development of HCG early in the disease process to identify a subgroup of PBC patients who could most likely benefit from surveillance.

\section{Patients and methods}

The Dutch Multicenter PBC Study is a multicenter prospective cohort study of PBC patients. The primary goal was to analyze long-term prognosis, in particular survival and causes of death, in patients treated with UDCA. Inclusion started in January 1990 and follow-up data until April 2007 were analyzed [1]. A definite diagnosis of PBC was made in the presence of two major plus two minor criteria or one major plus four minor criteria. Major criteria were antimitochondrial antibodies titer greater than or equal to $1: 20$ and compatible liver biopsy, whereas minor criteria were pruritus, jaundice, alkaline phosphatase (ALP) greater than or equal to twofold the upper limit of normal (ULN), serum IgM more than $2.8 \mathrm{~g} / \mathrm{l}$, and positive Schirmer test [13]. Available liver biopsies were reviewed according to Ludwig $e t$ al. [14]. Exclusion criteria were the earlier or current treatment with UDCA, aged above 75 years, pregnancy, evidence of extra hepatic biliary disease, autoimmuneoverlap syndrome or use of immunosuppressive drugs, concomitant disorders limiting life expectancy, and decompensated PBC, defined as Child-Pugh class B or $\mathrm{C}$ cirrhosis. Patients were eligible irrespective of the interval between diagnosis and inclusion. After inclusion, patients started with the UDCA therapy of $13-15 \mathrm{mg} / \mathrm{kg} /$ day [15]. Follow-up data with respect to HCC, other complications, liver transplantation (LTX), and (causes of) death were collected at 3-month intervals in the first year and at yearly intervals thereafter. At each visit general physical examination and laboratory studies including bilirubin, albumin, ALP, aspartate aminotransferase (AST), alanine aminotransferase, albumin, immunoglobulin $G$, immunoglobulin $\mathrm{M}$, and total cholesterol were performed. Liver tests were reported as the ratio of the test result to the ULN or lower limit of normal for the laboratory test.

Information on the causes of death was retrieved from hospital case records. In a limited number of cases, for instance if patients were lost to follow-up, family doctors or other hospitals were contacted.

The cohort study started well before the first consensus guidelines on HCC screening appeared. Therefore, with respect to screening liver imaging studies and measurement of fetoprotein levels were performed according to the policy of the individual participating hospitals.

\section{Definitions and statistical analysis}

Survival was analyzed as transplantation-free survival (end-points: LTX and death). Liver-related death was defined as: death because of liver failure or HCG, or death occurring within 2 months of an episode of variceal bleeding, spontaneous bacterial peritonitis, hepatorenal syndrome, or hepatic encephalopathy. Patients lost to follow-up were censored at the time of their last visit. Survival was compared with the survival of the Dutch population, matched for sex, age, and time of observation. Data for the Dutch population were derived from an official demographic database. For time-to-event analysis, we applied the Kaplan-Meier method and compared groups using the log-rank test.

Possible risk factors for HCC at diagnosis were evaluated using univariate and multivariate Cox regression analysis. We calculated the estimated risk to develop HCG based on the Silveira model. On the basis of serum bilirubin and albumin values the following prognostic classes were defined: early PBC (both bilirubin and albumin normal), moderately advanced PBC (one parameter normal), or advanced PBC (both parameters abnormal) [16]. Further, based on Mayo Risk Scores, the disease was classified 
as low risk (score $<4.5$ ) or high-risk (score $\geq 4.5$ ) [17]. Portal hypertension was defined as presence of ascites and/or a history of variceal bleeding. Biochemical response to UDCA treatment was defined as normal bilirubin and albumin concentrations after a 1-year treatment with UDCA when one or both parameters were abnormal before treatment, or as normal bilirubin or albumin concentration after treatment when both were abnormal at entry. Patients with persistently normal levels were also regarded as responders to therapy [1].

The occurrence of events during follow-up, including HCC, LTX, and death, was expressed as the cumulative incidence (CI) and the annual incidence (AI). CI represents the CI or total incidence over the complete follow-up period, whereas AI represents the mean AI.

A $P$ value of less than 0.05 (two-tailed) was considered statistically significant. Statistical package SPSS 15.0 (SPSS Inc., Chicago, Illinois, USA) was used to perform analyses.

\section{Results \\ Patients}

The baseline patient characteristics are summarized in Table 2. The study population comprised 375 (89\% female) patients, with a mean age of 54.7 years, who were followed for a median period of 9.7 (range: $1-17.3$ ) years. Sixteen (4\%) patients were lost to follow-up. After inclusion, the total number of study visits with collection of laboratory and clinical data was 4776; the average number of data collections was nine per patient. The majority of patients (60\%) suffered from early PBC. The available liver biopsies [65\% $(n=161)]$ were histologically classified as Ludwig stage I or II disease.

\section{Table 2 Baseline patient characteristics $(n=375)$}

\begin{tabular}{lc}
\hline Age (years; mean \pm SD) & $54.7 \pm 10.9$ \\
Sex (male/female) & $40(11 \%) / 335(89 \%)$ \\
AMA + & $350(93 \%)$ \\
ALP (ULN, range) & $2.7(0.4-13.0)$ \\
AST (ULN, range) & $1.6(0.3-7.4)$ \\
ALT (ULN, range) & $2.0(0.3-12.1)$ \\
Bilirubin (ULN, range) & $0.7(0.1-5.6)$ \\
Albumin (LLN, range) & $1.1(0.3-7.2)$ \\
Igm (ULN, range) & $1.9(0.2-17.5)$ \\
Igg (ULN, range) & $1.0(0.1-2.9)$ \\
Mayo Risk Score (mean \pm SD) & $4.1 \pm 1.0$ \\
Histological stage & \\
I & $69(18 \%)$ \\
II & $92(25 \%)$ \\
III & $52(14 \%)$ \\
IV & $34(9 \%)$ \\
Unknown & $128(34 \%)$ \\
Prognostic class & \\
Early PBC & $225(60 \%)$ \\
Moderately advanced PBC & $95(25 \%)$ \\
Advanced PBC & $34(9 \%)$ \\
Not available & $21(6 \%)$ \\
\hline
\end{tabular}

ALP, alkaline phosphatase; ALT, alanine aminotransferase; AMA, antimitochondrial antibodies; AST, aspartate amino transferase; lgG, immunoglobulin G; IgM, immunoglobulin M; LLN, lower limit of normal; PBC, primary biliary cirrhosis; SD, standard deviation; ULN, upper limit of normal.

\section{Survival}

Compared with a sample of the general Dutch population, transplantation free survival of our PBC cohort was significantly lower $(P<0.001)$. Survival of the total PBC cohort was $67 \%$, for the standardized Dutch population whereas the 15 -years survival rate was $80 \%$.

The incidence of nonliver related death in our cohort was comparable to that of the general population and the difference in survival can be explained by liver related causes, assuming liver related death and nonliver related death are independent events (Fig. 1).

\section{Causes of death}

During follow-up 69 out of 375 (18\%) patients died and $22(6 \%)$ underwent LTX. The number of deaths and transplantations was 34 in patients with early $\mathrm{PBC}, 35$ in moderately advanced PBC, and 22 in advanced PBC. Causes of death were mainly nonliver related for patients with early PBC: in this subgroup only $18 \%$ (six out of 34 ) of the patients died from liver related causes or underwent LTX. Liver related death and LTX occurred relatively more often in moderately advanced and advanced PBC: $63 \%$ (22 out of 35 ) and 91\% (20 out of 22), respectively. The AI of liver-related death and transplantation was $0.3,2.4$, and $8.8 \%$ for early, moderately advanced, and advanced $\mathrm{PBC}$, respectively (Table 3). HCC accounted for 33\% (two out of six) of liver-related causes of death in early $\mathrm{PBC}$, for $27 \%$ (six out of 22) in moderately advanced PBC, and for $5 \%$ (one out of 20) in advanced PBC. The corresponding AI were $0.1,0.6$, and $0.4 \%$, respectively (Table 3 ).

Fig. 1

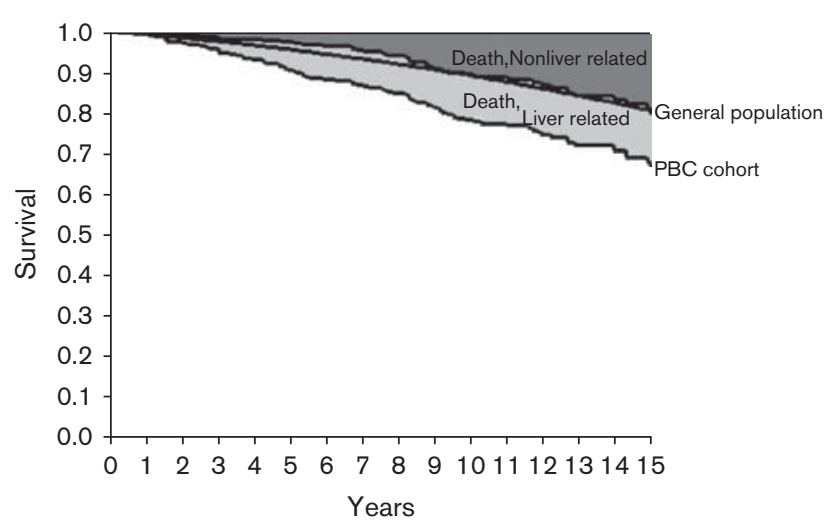

Survival of the cohort of 375 patients with primary biliary cirrhosis (PBC) compared with a matched sample of the Dutch population. Survival of patients with PBC was clearly lower than in the general population. The excess mortality in PBC is attributable to liver-related causes of death (light gray area). The incidence of nonliver related death (dark gray area) was comparable for both populations (the line between liver related death and nonliver related death was calculated, assuming that liver related death and nonliver related death are not related). 


\section{Hepatocellular carcinoma cases}

During follow-up nine cases of HCG were diagnosed (Table 4). Seven of these patients were female, the mean age at the diagnosis of $\mathrm{PBC}$ was $57 \pm 9$ years, and at the HCC diagnosis of $66 \pm 11$ years. At HCC diagnosis $33 \%$ (three out of nine) of the patients were aged above 70 years. The time from PBC diagnosis to the development of HCC was $16 \pm 5$ [mean \pm standard deviation $(\mathrm{SD})]$ years.

Diagnosis was made by the characteristic findings on MRI or computed tomography imaging in combination with markedly elevated (and rising) $\alpha$-fetoprotein levels. Four out of the nine cases with HCC diagnosis were confirmed by histology of needle biopsies $(n=2)$, surgical explant specimen $(n=1)$, or autopsy material $(n=1)$.

At PBC diagnosis the Mayo Risk Score was greater than or equal to 4.5 in $78 \%$ of the patients, at HCC diagnosis this was $100 \%$. Initially, liver histology showed Ludwig stage III or IV in four out of seven cases, but at the time of HCC diagnosis all nine patients had progressed to stage III or IV.

Three patients were diagnosed with multifocal HCC. The mean diameter of the largest tumor was $42 \pm 28 \mathrm{~mm}$ (SD). At the time of HCC diagnosis $\alpha$-fetoprotein levels were elevated in all cases, varying from three to 112 times the ULN.

Table 3 Causes of death in a cohort of 375 ursodeoxycholic acid-treated primary biliary cirrhosis patients

\begin{tabular}{|c|c|c|c|}
\hline & Early PBC $(n=225)$ & Moderately advanced PBC $(n=95)$ & Advanced PBC $(n=34)$ \\
\hline Age at entry (years; mean $\pm S D$ ) & $54 \pm 11$ & $55 \pm 11$ & $57 \pm 10$ \\
\hline Follow-up (months; mean $\pm S D$ ) & $124 \pm 50$ & $118 \pm 55$ & $80 \pm 56$ \\
\hline Liver related death/transplantation $(n)(\mathrm{Al})$ & $6(0.3)$ & $22(2.4)$ & $20(8.8)$ \\
\hline Liver failure & $4(0.2)$ & $16(1.7)$ & $20(8.8)^{a}$ \\
\hline $\mathrm{HCC}$ & $2(0.1)$ & $6(0.6)$ & - \\
\hline Nonliver related death $(n)(\mathrm{Al})$ & $28(1.2)$ & $13(1.4)$ & $2(0.9)$ \\
\hline Cardio-respiratory disease & $11(0.5)$ & $2(0.2)$ & $1(0.4)$ \\
\hline Cerebrovascular accident & $3(0.1)$ & $4(0.4)$ & - \\
\hline Nonhepatic malignancy & $8(0.3)$ & $1(0.1)$ & - \\
\hline Other causes & $6(0.3)$ & $6(0.6)$ & $1(0.4)$ \\
\hline Alive at end of study $(n)$ & 180 & 56 & 11 \\
\hline Age at the time of death (years; mean $\pm S D$ ) & $72(9)$ & $63(13)$ & $65(10)$ \\
\hline
\end{tabular}

HCC was detected in autopsy material.

Al, annual incidence; HCC, hepatocellular carcinoma; PBC, primary biliary cirrhosis; SD, standard deviation.

${ }^{\mathrm{a}}$ One patient died from liver failure.

Table 4 Details of nine patients with primary biliary cirrhosis who developed hepatocellular carcinoma

\begin{tabular}{|c|c|c|c|c|c|c|c|c|c|c|}
\hline Case & 1 & 2 & 3 & 4 & 5 & 6 & 7 & 8 & 9 & Total \\
\hline Female sex & No & No & Yes & Yes & Yes & Yes & Yes & Yes & Yes & $78 \%$ \\
\hline Age at diagnosis PBC (years) & 56 & 57 & 42 & 47 & 48 & 62 & 58 & 37 & 39 & $50 \pm 9$ \\
\hline Age at diagnosis HCC (years) & 76 & 68 & 50 & 66 & 63 & 78 & 78 & 50 & 60 & $66 \pm 11$ \\
\hline Interval diagnosis $\mathrm{PBC}-\mathrm{HCC}$ (years) & 20 & 11 & 7 & 19 & 14 & 16 & 20 & 13 & 21 & $16 \pm 5$ \\
\hline \multicolumn{11}{|l|}{ Mayo Risk Score } \\
\hline At diagnosis of PBC & 4.6 & 4.8 & 5.6 & 3.7 & 6.1 & 4.9 & 5.2 & 4.6 & 4.4 & $4.9 \pm 0.7$ \\
\hline At diagnosis of $\mathrm{HCC}$ & - & 5.5 & 5.7 & 4.9 & 9.8 & 9.0 & 8.0 & - & 5.8 & $7.0 \pm 5.8$ \\
\hline Histological stage PBC & & & & & & & & & & $3.4 \pm 1.3$ \\
\hline At diagnosis PBC & - & III & III & II & III & NA & II & II & IV & \\
\hline At diagnosis $\mathrm{HCC}$ & IV & III & IV & IV & IV & III & IV & IV & IV & $3.8 \pm 0.4$ \\
\hline Portal hypertension & No & Yes & Yes & Yes & Yes & Yes & Yes & NA & Yes & $88 \%$ \\
\hline History blood transfusion & No & Yes & No & No & No & No & No & NA & No & $13 \%$ \\
\hline Body mass index & 22 & 27 & 22 & 25 & 19 & 30 & 26 & 30 & 22 & $25 \pm 4$ \\
\hline Other liver disease & No & No & No & No & No & Yes $^{a}$ & No & No & No & $11 \%$ \\
\hline Nodules & 2 & 1 & 1 & 2 & 1 & 1 & 2 & 1 & 1 & $1.3 \pm 0.5$ \\
\hline Diameter $(\mathrm{mm})^{\mathrm{b}}$ & 80 & - & 5 & 37 & 24 & 25 & 68 & 75 & 25 & $42 \pm 28$ \\
\hline \multicolumn{11}{|l|}{ AFP levels (ULN) } \\
\hline At diagnosis PBC & - & 0.2 & 0.6 & - & 0.8 & - & 0.7 & - & 0.7 & $0.6 \pm 0.2$ \\
\hline At diagnosis $\mathrm{HCC}$ & 55.6 & 56.7 & 12.9 & 112.1 & 11.2 & 3.3 & 23.1 & NA & 72.3 & $42 \pm 40$ \\
\hline Predicted risk for $\mathrm{HCC}(\%)^{\mathrm{c}}$ & 5.9 & 46.7 & 5.6 & 15.6 & 15.6 & 64.8 & 64.8 & - & 15.6 & $28 \pm 24$ \\
\hline \multicolumn{11}{|l|}{ Prognostic class $^{d}$} \\
\hline At diagnosis PBC & 2 & 1 & 3 & 1 & 2 & 2 & 2 & 2 & 2 & $1.9 \pm 0.6$ \\
\hline At diagnosis $\mathrm{HCC}$ & 3 & 3 & 3 & NA & 3 & 3 & 3 & NA & 3 & 3 \\
\hline Responder to UDCA & No & Yes & No & Yes & No & No & No & No & No & $22 \%$ \\
\hline Treatment & None & None & LTX & RFA & None & None & None & None & PEI & - \\
\hline Status & Dead & Dead & Alive & Dead & Dead & Dead & Dead & Dead & Dead & - \\
\hline Survival (months) & 10 & 14 & 133 & 23 & 0 & 1 & 35 & 23 & 9 & $13 \pm 12$ \\
\hline
\end{tabular}

AFP, $\alpha$-fetoprotein; HCC, hepatocellular carcinoma; LTX, liver transplantation; NA, not available; PBC, primary biliary cirrhosis; PEI, percutaneous ethanol injection; RFA, radio frequency ablation; UDCA, ursodeoxycholic acid; ULN, upper limit of normal.

${ }^{\mathrm{a}}$ Earlier hepatitis $\mathrm{B}$, HBsag negative.

${ }^{b}$ Of the largest tumour nodule.

${ }^{\mathrm{c}}$ According to Silveira et al. [11].

$\mathrm{d}_{1}=$ early PBC, $2=$ moderately advanced PBC, $3=$ advanced PBC. 
The mean predicted probability to develop HCC according to the Silveira model, based on sex, age, presence of portal hypertension, and history of blood transfusion, was $28 \pm 24 \%$ (SD) [11].

According to pretreatment bilirubin and albumin levels, seven out of the nine HCC cases were categorized as moderately advanced or advanced PBC at diagnosis (78\%). All seven patients were nonresponders to treatment with UDCA, based on Rotterdam criteria.

According to the Paris [18] and Barcelona [19] criteria for response to UDCA treatment, one out of nine (11\%) and six out of nine $(67 \%)$, respectively, of our PBC patients with HCG were nonresponders. However, the two patients not responding according to the Rotterdam criteria were responders according to the Barcelona criteria. Thus, responders identified by one set of criteria are not identical to responders when other criteria are used, and the way biochemical response is defined is obviously of major importance when interpreting the results of this and comparable studies.

All but one patient died within 3 years of HCG diagnosis. One patient underwent liver transplantation and was alive for 133 months.

\section{Risk factors hepatocellular carcinoma}

To determine the risk factors for the development of HCC, univariate analysis was performed concerning the following baseline variables: age, sex, antimitochondrial antibodies, total serum bilirubin, serum albumin, ALP, alanine aminotransferase, AST, the presence or absence of cirrhosis and portal hypertension, Mayo Risk Score, and prognostic class (early/moderately advanced/advanced PBC). Bilirubin $(P=0.001)$, AST $(P=0.003)$, prognostic class $(P=0.017)$, and Mayo Risk Score $(P=0.004)$ were significantly associated factors. In multivariate analysis no factor remained statistically significant.

In univariate analysis, at 1 year after diagnosis, response to UDCA was significantly associated with the development of HCG $(P=0.001)$.

In the total population the AI of HCC was $0.2 \%$ and the CI was 2.4\% (nine out of 375) (Fig. 2a). The incidence of HCC was comparable for men and women $(P=0.32)$, for Ludwig stage I/II and III/IV $(P=0.98)$ and for the presence and absence of portal hypertension $(P=0.112)$. Mayo Risk Scores greater than or equal to 4.5 and moderately advanced $\mathrm{PBC}$ were significant risk factors for HCC (Fig. 2e and f). The highest incidence of HCC was found in patients who were nonresponders to UDCA treatment (Fig. 2g).

The AI of HCC in patients with cirrhosis $(n=86)$ was $0.2 \%$, in patients with Mayo Risk Scores greater than or equal to 4.5 was $(n=96) 1 \%$, in patients with moderately advanced PBC was $(n=95) 0.7 \%$, and in patients not responding to UDCA was $1.4 \%$. In patients with moderately advanced disease who failed to respond to UDCA $(n=48)$, the annual risk was $1.5 \%$.

In $78 \%$ of HCC cases the Mayo Risk Score was greater than or equal to 4.5. Hypothetically, when this criterion would have been used for initiating a screening program, and assuming $100 \%$ sensitivity of the program, the number needed to screen (NNS) to identify one case with HCG would have been 14. Similarly, the NNS in moderately advanced PBC cases would have been 16, in UDCA nonresponders 11 , and in moderately advanced PBC and UDCA nonresponse eight.

\section{Discussion}

This cohort study consisting of 375 UDCA treated PBC patients found a total incidence of HCG of $2.4 \%$ after a median follow-up of 9.7 years. The AI of HCC was $0.2 \%$ and this is the lowest reported risk of HCC in PBC. However, a comparable incidence of HCC in PBC was found by two other studies [2,6]. Nineteen percent of liver related death was secondary to HCC and this was responsible for $30 \%$ of liver related mortality in patients with early or moderately advanced PBC. Thus, although the absolute number of cases with HCC was only nine, HCC was a significant cause of death secondary to liver disease. The most important risk factor for future development of HCC in this study was the nonresponse to UDCA treatment, which was noted in seven out of the nine HCG cases. In the subgroup of patients not responding to UDCA the incidence of HCC was 9\% after 10 years and $20 \%$ after 15 years. The $a$ posteriori calculated NNS to identify a case of HCC in this population was 11. In the group of patients with advanced PBC only one case with HCC was observed, this was an incidental finding at autopsy of patients who died from liver failure. The low incidence of HCC in this subgroup might be explained by the severity of the disease leading to liver-related death and liver transplantation preceding potential development of HCC.

Although this study included a relatively large population followed prospectively during a substantial time period, the absolute number of HCC cases was low. Therefore, the results should be interpreted cautiously, and our finding that nonresponse to UDCA is a main risk factor for HCC awaits confirmation by other studies. In this study the screening strategy was left to the individual participating centers. In theory a uniform, standardized program could have been more effective in identifying cases with HCC. It seems unlikely, however, that this would have resulted in identifying substantially more cases, given the strong association between HCC and clinical symptoms, albeit at variable points in time of its natural history.

This study did not confirm the major prognostic importance of cirrhosis for future HCC. It should be noted, however, that histological staging at entry was possible in only two-thirds of the population. Possibly this 
Fig. 2
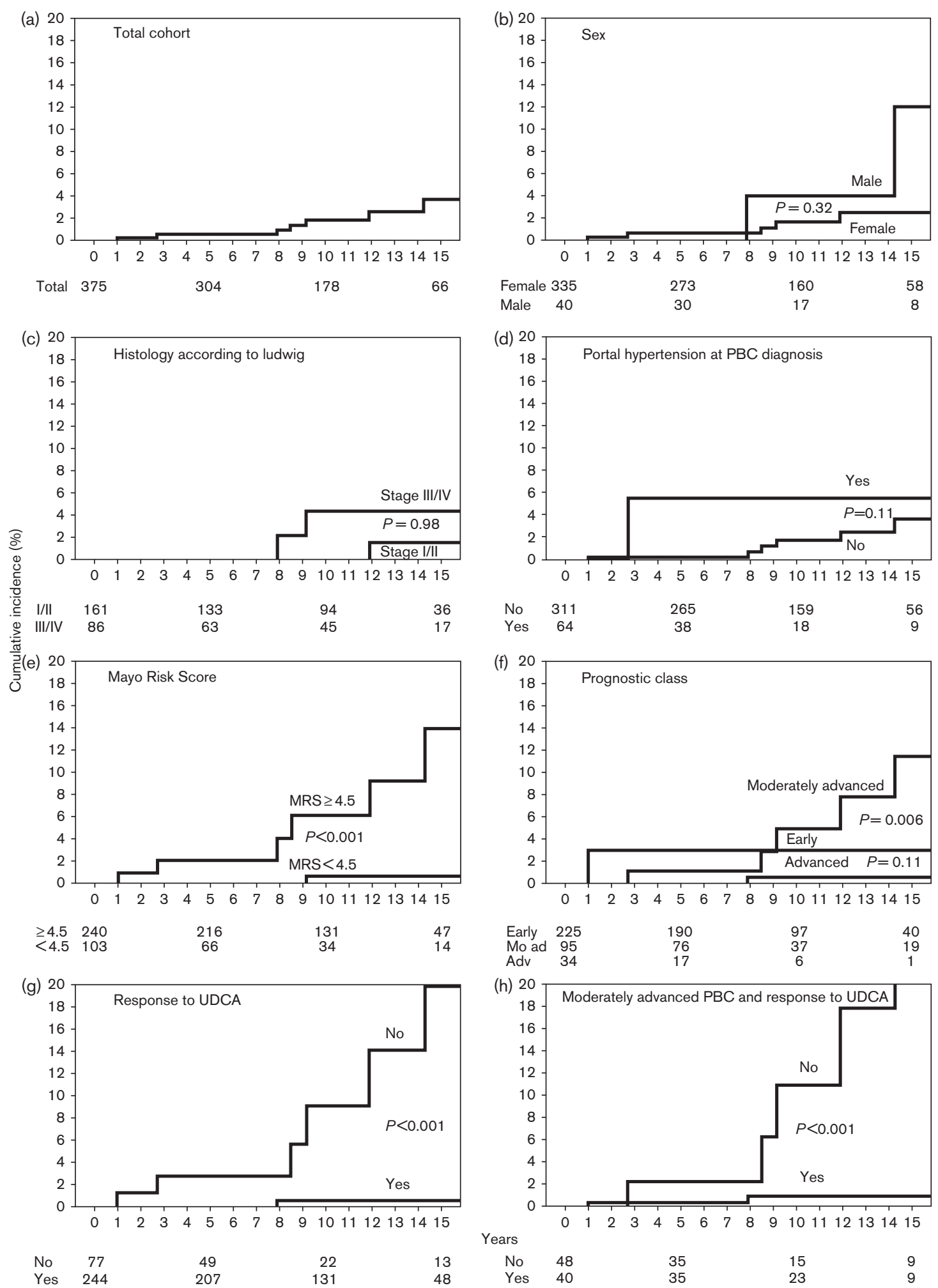

Kaplan-Meier plots showing the cumulative incidence of hepatocellular carcinoma in the total group of patients with primary biliary cirrhosis (PBC) (a) and in subgroups of patients according to sex (b), histological stage (c), presence of portal hypertension (d), Mayo Risk Score (MRS) (e), prognostic class at the time of PBC diagnosis ( $\mathrm{f}$ ), response to ursodeoxycholic acid (UDCA) after 1-year of treatment $(\mathrm{g})$ and the combination of moderately advanced PBC and response to UDCA (h). The number of patients at risk is shown under the $x$-axis. 
may have resulted in lack of power for cirrhosis in the multivariate Cox regression analysis. Therefore, the results of this study should not be interpreted as providing proof for cirrhosis not being a major risk factor for HCG.

A potential major advantage of using nonresponse to UDCA for selecting patients for screening procedures for HCG is that such patients can be recognized early, that is after 1-year treatment with UDCA. In addition, the necessary data are easily available and do not require particular (invasive) diagnostic procedures such as liver biopsy or gastrointestinal endoscopy. We have found earlier that nonresponders in comparison with responders had higher pretreatment serum levels of bilirubin, ALP, AST, higher Mayo Risk Scores, and histologically more advanced disease. Survival for nonresponders was significantly worse than for responders [1]. Hypothetically, ongoing inflammatory activity and cholestasis superimposed on significant liver fibrosis or cirrhosis are the most important drivers of carcinogenesis in UDCA nonresponsive patients. For the time being, however, it remains speculative as to which biological mechanism could explain the high cancer risk in UDCA nonresponders, also considering the significantly lower or lacking prognostic significance of cirrhosis, portal hypertension, and Mayo Risk Score.

Two out of the nine patients developing HCG were responders to UDCA. Both patients had biochemically nonadvanced disease at entry, as reflected by normal serum bilirubin and albumin levels. We were unable to identify particular factors posing these patients at an increased risk for HCC.

Obviously, further studies are required to confirm the prognostic significance of nonresponse to UDCA according to our criteria and to determine whether nonresponse according to other, in particular the Barcelona [19], criteria is a risk factor for HCC.

We could not confirm the usefulness of the Silveira risk model for HCC [20]. This model was developed using a case-control design and found that older age, male sex, history of blood transfusion, and any sign of portal hypertension indicated a high risk of HCC. Our patients with HCC were comparable with the Mayo clinic patients at the time of HCG diagnosis with respect to age, BMI, alcohol consumption, histological stage at PBC diagnosis, presence of portal hypertension, and Mayo Risk Score. However, the duration from PBC diagnosis until the development of HCC was significantly longer in our population compared with the Mayo clinic patients (93 \pm 13 vs. $192 \pm 60$ months), the proportion of male patients was lower (41 vs. 22\%), and our patients often received less blood transfusions (59 vs. 13\%). Possible explanations for these diverging results are a retrospective versus a prospective study design, geographical differences, and a potential protective or delaying effect of UDCA in the development of HCC. A protective effect of UDCA was also suggested by Jackson et al. [2] who reported a three-fold increased risk for HCC in treated PBC patients versus an eight-fold increased risk in untreated patients in comparison with the general population. Another study including treated and nontreated patients found that UDCA was not independently associated with the development of HCC [3].

According to the American Association for the Study of Liver Diseases and the European Association for the Study of the Liver practice guidelines surveillance programs in patients with cirrhosis should be considered when the risk of HCC is at least $1.5 \%$ per year $[12,21]$. The American Association for the Study of Liver Diseases guideline proposed screening in PBC patients with cirrhosis based on earlier findings of a risk for HCC that was comparable with that in hepatitis $\mathrm{C}$ cirrhosis [7]. We found an AI of HCC in individuals with cirrhosis of only $0.2 \%$ and this would clearly argue against screening in this population. Several other studies also reported a risk for HCC lower than $1.5 \%$ per year in patients with advanced histological stages $[4,9,5]$. In our study the only population carrying the proposed minimal $1.5 \%$ annual risk for HCC was the group of patients with moderately advanced PBC who failed to respond to UDCA. However, three out of our nine HCC cases would not have been selected for screening according to these criteria. A slightly lower annual HCG risk of $1.4 \%$ was established for patients not responding to UDCA. Our data, therefore, suggest that the population most likely to benefit from screening would be patients who fail to respond to UDCA.

In conclusion, this multicenter prospective study found a relatively low risk for HCC in patients who were all treated with UDCA for a long-term. We could not confirm the prognostic importance of male sex, cirrhosis, portal hypertension, and a history of blood transfusion. The strongest risk factor for HCC in our population was the absence of biochemical response after 1-year treatment with UDCA. In this subgroup the 15-year incidence of HCC was 20\% and the NNS was 11.

Further studies are required to confirm that the simple criterion of nonresponse to UDCA treatment is superior to cirrhosis as the criterion for selecting individuals with PBC for screening programs.

\section{Acknowledgements}

The following members of the Dutch Multicenter PBC Study Group participated in the study: M.J. Wagtmans (Almere), R.J. Robijn (Apeldoorn), R.A. de Vries (Arnhem), P.J.J. Leeuwerik, M. Rasica (Bergen op Zoom), A.E.F. Lückers (Boxmeer), M.C.M. Rijk, G.J. Ras (Breda), C.T.B.M van Deursen (Brunssem), E.W. van der Hoek, J.W. de Bruijne, I.P. van Munster, Th.J.M. van Ditzhuijzen (Den Bosch), M.H.M.G. Houben, R.M. Valentijn, S.D.J. van der Werf (Den Haag), F. ter Borg (Deventer), 
R. Beukers, W. Lesterhuis, A.C.M. van Vliet, H.H. Ponssen, R.F. Eichhorn, W. van de Vrie (Dordrecht), B.J.M. Witteman, J. van Aken (Ede), A. Stronkhorst, P.J. Boekema (Eindhoven), M.J. Kerbert-Dreteler (Enschede), R. de Kan (Goes), E.B. Haagsma (Groningen), J.N. Groen (Harderwijk), T.G. Tan, F.N.R. van Berkum (Hengelo), J.C.Thijs (Hoogeveen), R. Zwertbroek, T.A. Grool (Hoorn), B. van Hoek (Leiden), G.H. Koek (Maastricht), P.N.M van Hees, R.W. de Koning, R.G.L. de Sévaux, W.A. de Boer (Oss), P. Biemond (Roosendaal), A.J.P. van Tilburg, J.W. den Ouden, F.J.G.M. Kubben (Rotterdam), R.N.M. Zeijen (Schiedam), L.G.J.B. Engels, B.J.Looij (Sittard), F.H.J. Wolfhagen (Tilburg), K.J. van Erpecum, F.P. Vleggaar (Utrecht), A.A. Tanis (Vlissingen), J.G.S.Breed (Weert), B.C.A.J. Loffeld (Woerden), O.A. van Dobbenburgh (Zutphen), J. Lambert (Zwolle). H.R.V.B. and E.M.M.K. received an unrestricted Grant from Zambon BV, Amersfoort. There is no conflict of interest to declare.

\section{References}

1 Kuiper EM, Hansen BE, De Vries RA, Den Ouden-Muller JW, Van Ditzhuijsen TJ, Haagsma EB, et al. Improved prognosis of patients with primary biliary cirrhosis that have a biochemical response to ursodeoxycholic acid. Gastroenterology 2009; 136:1281-1287.

2 Jackson H, Solaymani-Dodaran M, Card TR, Aithal GP, Logan R, West J. Influence of ursodeoxycholic acid on the mortality and malignancy associated with primary biliary cirrhosis: a population-based cohort study. Hepatology 2007; 46:1131-1137.

3 Deutsch M, Papatheodoridis GV, Tzakou A, Hadziyannis SJ. Risk of hepatocellular carcinoma and extrahepatic malignancies in primary biliary cirrhosis. Eur J Gastroenterol Hepatol 2008; 20:5-9.

4 Farinati F, Floreani A, De MN, Fagiuoli S, Naccarato R, Chiaramonte M. Hepatocellular carcinoma in primary biliary cirrhosis. $J$ Hepatol 1994; 21:315-316.

5 Jones DE, Metcalf JV, Collier JD, Bassendine MF, James OF. Hepatocellular carcinoma in primary biliary cirrhosis and its impact on outcomes. Hepatology 1997; 26:1138-1142.

6 Nijhawan PK, Therneau TM, Dickson ER, Boynton J, Lindor KD. Incidence of cancer in primary biliary cirrhosis: the Mayo experience. Hepatology 1999; 29:1396-1398.
7 Caballeria L, Pares A, Castells A, Gines A, Bru C, Rodes J. Hepatocellular carcinoma in primary biliary cirrhosis: similar incidence to that in hepatitis C virus-related cirrhosis. Am J Gastroenterol 2001; 96:1160-1163.

8 Shibuya A, Tanaka K, Miyakawa H, Shibata M, Takatori M, Sekiyama K, et al. Hepatocellular carcinoma and survival in patients with primary biliary cirrhosis. Hepatology 2002; 35:1172-1178.

9 Findor J, He XS, Sord J, Terg R, Gershwin ME. Primary biliary cirrhosis and hepatocellular carcinoma. Autoimmun Rev 2002; 1:220-225.

10 Floreani A, Baragiotta A, Leone MG, Baldo V, Naccarato R. Primary biliary cirrhosis and hepatitis $C$ virus infection. Am J Gastroenterol 2003; 98:2757-2762.

11 Silveira MG, Suzuki A, Lindor KD. Surveillance for hepatocellular carcinoma in patients with primary biliary cirrhosis. Hepatology 2008; 48:1149-1156.

12 Bruix J, Sherman M. Management of hepatocellular carcinoma. Hepatology 2005; 42:1208-1236.

13 Taal BG, Schalm SW, Ten Kate FW, Hermans J, Geertzen RG, Feltkamp BE. Clinical diagnosis of primary biliary cirrhosis: a classification based on major and minor criteria. Hepatogastroenterology 1983; 30:178-182.

14 Ludwig J, Dickson ER, McDonald GS. Staging of chronic nonsuppurative destructive cholangitis (syndrome of primary biliary cirrhosis). Virchows Arch A Pathol Anat Histol 1978; 379:103-112.

15 Van Hoogstraten HJ, Hansen BE, Van Buuren HR, Ten Kate FJ, Van Berge-Henegouwen GP, Schalm SW. Prognostic factors and long-term effects of ursodeoxycholic acid on liver biochemical parameters in patients with primary biliary cirrhosis. Dutch Multi-Centre PBC Study Group. J Hepatol 1999; 31:256-262.

16 Ter Borg PC, Schalm SW, Hansen BE, Van Buuren HR. Prognosis of ursodeoxycholic acid-treated patients with primary biliary cirrhosis. Results of a 10-year cohort study involving 297 patients. Am J Gastroenterol 2006; 101:2044-2050.

17 Dickson ER, Grambsch PM, Fleming TR, Fisher LD, Langworthy A. Prognosis in primary biliary cirrhosis: model for decision making. Hepatology 1989; 10:1-7.

18 Corpechot C, Abenavoli L, Rabahi N, Chretien Y, Andreani T, Johanet $\mathrm{C}$, et al. Biochemical response to ursodeoxycholic acid and long-term prognosis in primary biliary cirrhosis. Hepatology 2008; 48:871-877.

19 Pares A, Caballeria L, Rodes J. Excellent long-term survival in patients with primary biliary cirrhosis and biochemical response to ursodeoxycholic acid. Gastroenterology 2006; 130:715-720.

20 Suzuki A, Lymp J, Donlinger J, Mendes F, Angulo P, Lindor K. Clinical predictors for hepatocellular carcinoma in patients with primary biliary cirrhosis. Clin Gastroenterol Hepatol 2007; 5:259-264.

21 Bruix J, Sherman M, Llovet JM, Beaugrand M, Lencioni R, Burroughs AK, et al. Clinical management of hepatocellular carcinoma. Conclusions of the Barcelona-2000 EASL conference. European Association for the Study of the Liver. J Hepatol 2001; 35:421-430. 